\title{
Online Quality Assurance of Microchannels in Roll- to-Roll by Optical Coherence Tomography
}

\author{
Janne Lauri \\ Optoelectronics and Measurement \\ Techniques Research Unit \\ University of Oulu \\ Oulu, Finland \\ janne.lauri@oulu.fi \\ Tapio Fabritius \\ Optoelectronics and Measurement \\ Techniques Research Unit \\ University of Oulu \\ Oulu, Finland \\ tapio.fabritius@oulu.fi
}

\author{
Christina Liedert \\ VTT, Technical Research Centre of \\ Finland \\ Oulu, Finland \\ christina.liedert@vtt.fi
}

\author{
Ralph Liedert \\ VTT, Technical Research Centre of \\ Finland \\ Oulu, Finland \\ ralph.liedert@vtt.fi
}

\begin{abstract}
Roll-to-roll (R2R) process is an emerging technology to manufacture printed electronics, microfluidics, biochemical sensors etc. The requirements for high quality and small tolerances at the manufacturing phase are of the essence for such products. To verify the quality and guarantee the high production yield, high speed, non-destructive testing methods are needed. In this paper, optical coherence tomography (OCT) device is used at the R2R-line to measure online hot embossed microchannel structures at speed of $1 \mathrm{~m} / \mathrm{min}$, which is typical for the hot embossing process. The channel's width and shape are determined along the web. The applicability of OCT for topography measurements is demonstrated in an actual R2R environment.
\end{abstract}

Keywords-non-destructive testing, surface topography, online measurement, profilometer

\section{INTRODUCTION}

Microfluidics is an emerging technology that can be used to control flow of fluids, e.g. biological samples and reagents, in very small volumes. Various functionalities, such as mixing, moving a sample, filtering and particle labeling, can be implemented in microfluidics to replace laborious and time-consuming laboratory procedures. A huge impact of the microfluidics is expected to be in point-of-care testing by providing a platform for low cost biosensors. To guarantee a reliability of the biosensor, tolerances for microfluidic manufacturing are demanding, especially when low cost is required.

One of the potential techniques to mass manufacture low cost microfluidic channels is to use roll-to-roll (R2R) processes e.g. R2R hot embossing. In hot embossing, a typical web speed is $0.5-1.5 \mathrm{~m} / \mathrm{min}$ and feature sizes are in a range from few tens to hundreds of micrometers. The most critical parameter to be monitored during the process is the microchannel's shape (including a width and a depth), which is optimized by changing a temperature, a web speed and an embossing pressure. To obtain continuous feedback and to monitor the embossing quality, there is a need for noncontact imaging methods that are able to cover a topography as well as an inner structure of a large area sample with a micrometer precision. A R2R-line can produce hundreds of microchannels in a minute (depends on web width and channel size). To fulfill the requirement for a low cost and reliable biosensor, a yield must be high and this sets high demands for a fast online quality control.

J.L is partially supported by European Regional Development Fund (project: PrintoCent10, grant no. A73286). T.F is partially supported by Academy of Finland's FIRI funding (grant no. 320017).
Many optical techniques based on wavelength scanning interferometry [1], multi-wavelength digital holography [2], structured light [3] have been applied to measure surface topography. Typically, in these techniques multiple frames are taken at different wavelengths or phases to calculate a single surface topography image. The achieved areal measurement speed is often not applicable for surface characterization of a moving sample due to the motion blur and vibrations occurring during the wavelength sweep or the phase scan. Chromatic aberration of focused light [4] has recently demonstrated its potential in high-speed surface imaging and inner structure of transparent layers [5].

A 3D imaging technique, called optical coherence tomography (OCT), has emerged as a powerful tool in nondestructive testing of various types samples including microfluidics [6]. OCT is based on low coherence interferometry, where micron-scale axial resolution is obtained by exploiting a coherence gating and a broadband light source [7]. A single depth scan is called A-scan and by lateral scanning a probing beam, cross-sectional images, Bscans, are obtained. Aside from its main application field in biomedical imaging, especially in ophthalmology, OCT has been applied e.g. in microfluidics [8,9], printed electronics [10], displays [11] and LEDs [12]. In addition, it has been recently demonstrated in quality measurements of printed electronics in a simulated R2R-line [13].

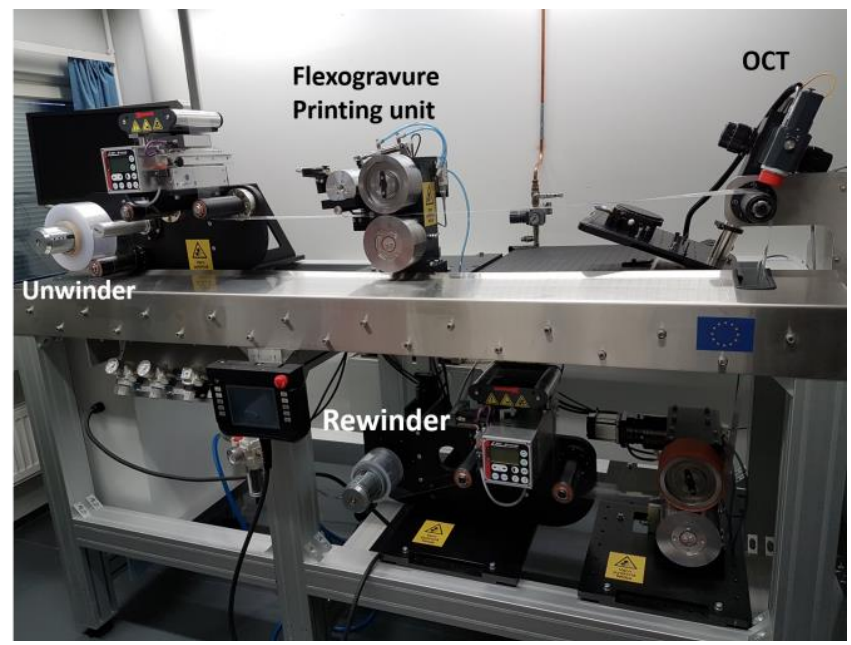

Fig. 1. A pilot R2R-line (OPK-2017) with the OCT installed over the guiding roll. The flexogravure printing unit is not used during measurements. 


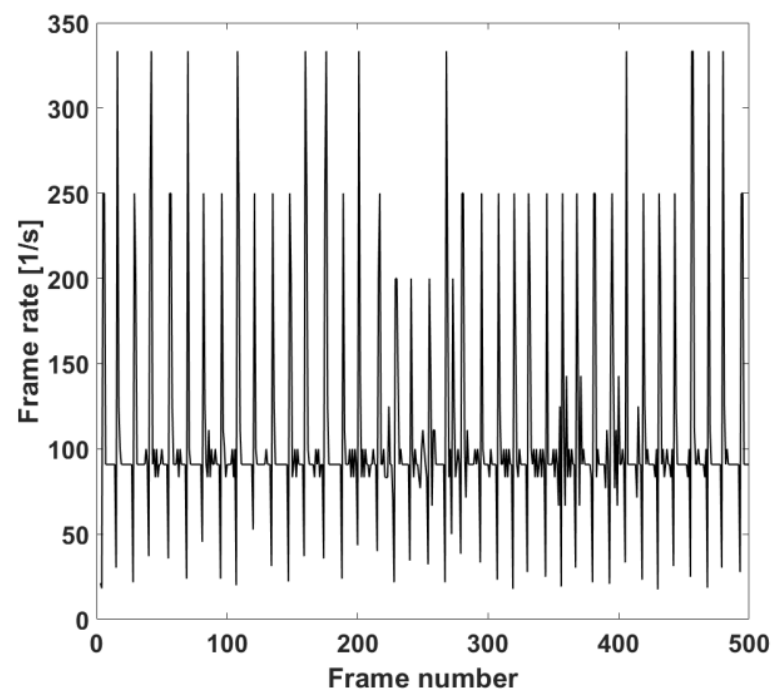

Fig. 2. Frame rate for 1000 A-scans per frame calculated from the elapsed time between the sequential frames. Reasons for the periodical variations in the frame rate is not known.

In this paper, OCT is used online in the actual R2R-line to measure a quality of hot embossed microchannels. A channel width is determined and shape is resolved. Limitations of the used OCT are discussed.

\section{MATERIAL AND METHODS}

\section{A. Sample and R2R unit}

The OCT device was installed at the custom made R2Rline (OPK-2017) shown in Fig. 1. The measurement location is over the guiding roll to reduce extensive vibration of the web. Due the lack of hot embossing unit in OPK-2017, the microchannels were fabricated at VTT (Technical Research Centre of Finland, Oulu, Finland) with the PIKO-pilot line. The microchannel structures were R2R hot embossed on thermoplastic polymethyl methacrylate (PMMA) foil (Plexiglass 99524, Evonik Röhm, Germany) with a web width of $200 \mathrm{~mm}$ and thickness of $375 \mu \mathrm{m}$. The width and height of the relief in the hot embossing roll were $200 \mu \mathrm{m}$ and $80 \mu \mathrm{m}$ respectively. The web was cutted to the width of $8 \mathrm{~cm}$ in order to fit it to the OPK-2017 line. The speed of the web for the surface measurements was set to $1 \mathrm{~m} / \mathrm{min}$, which was the same as in the hot embossing process.

\section{B. $O C T$}

A commercial OCT (Hyperion, Thorlabs Inc.) was used in the measurements of embossed microfluidic structure at the R2R-line. The axial (depth) and the lateral resolution of the system were $5.8 \mu \mathrm{m}$ (in air) and $8 \mu \mathrm{m}$ respectively. For the cross direction (CD) scanning, i.e. across the web, the maximal lateral scanning length of $(10 \mathrm{~mm})$ the system was utilized. Three sets, each consisting of 500 cross-sectional images (B-scans), were acquired at the web speed of 1 $\mathrm{m} / \mathrm{min}$. In addition, to estimate vibrations caused by the moving web, a reference measurement of the stopped web was taken. The B-scans consisted of 200, 500 and 1000 depth scans, so-called A-scans. As the A-scan acquisition rate is constant, the frame rate depends on the number of $\mathrm{A}$ scans in one frame. During a measurement, a time between frames was not completely stable (see Fig. 2.), thus an averaged time was used to determine the frame rate. For the $200 \mathrm{~A}$-scans the averaged frame rate was $183 \mathrm{~Hz}$ and the lateral step size in the CD-direction was $50 \mu \mathrm{m}$. For the 500 and $1000 \mathrm{~A}$-scans the frame rates were $123 \mathrm{~Hz}$ and $82 \mathrm{~Hz}$, and the lateral steps sizes $20 \mu \mathrm{m}$ and $10 \mu \mathrm{m}$ respectively. The resolutions in the web direction, so-called machine direction (MD), were $91 \mu \mathrm{m}, 136 \mu \mathrm{m}$ and $200 \mu \mathrm{m}$ from the highest frame rate to the lowest.

\section{Optical profilometer}

The optical profilometer (ContourGT-K, Bruker) was used for the reference measurements. The sample was cut from the web and measured offline. The measurement location is approximately the same as for the OCT but taken from different location along the web.

\section{Data processing}

To maximize speed of the OCT, the raw camera data is first buffered memory and saved to hard drive after the measurement. The raw data is than converted to intensity image with the original OCT-software. The intensity data was offline processed in Matlab (R2019a). Simple image processing algorithms were applied to keep computation time short for an online application purposes.

A non-telecentric scanning in the OCT system causes geometrical distortions to an image, e.g. flat surface appears to be convex or concave depending on the position of the scanning mirrors relative to the focal point of the objective lens $[14,15]$. Therefore, the intensity data is first corrected for the non-telecentric distortions in Matlab. For calibration, a flat mirror surface is measured and linearized. The obtained calibration curve is used to correct geometrical distortions. After the distortion correction, a region of interest, including only the surface of the PMMA-foil, is defined. The surface is segmented by finding maxima along the A-scans followed by a median filtering. The number and the widths of channels in the B-scan were defined by finding the largest differences in the neighboring depth pixel followed by Matlab peak detection routine ('findpeaks'). The processing times for 500 frames, (processor: Intel i7-4790) were $1.7 \mathrm{~s}, 3.2 \mathrm{~s}$ and $6.1 \mathrm{~s}$ (200, 500, 1000 A-scans/frame).

\section{RESULTS AND DISCUSSION}

Fig. 3 shows the outcome of the geometrical correction of the non-telecentric scanning. It should be noticed, that the geometrical distortions are depth dependent, thus the measured surface should be at similar depths as the calibration mirror surface. Here, the corrected PMMA surface becomes flat, however, there is a residual tilt due to a non-perpendicular scanning along the sample surface. For more accurate surface characterization e.g. finer details, more sophisticated numerical algorithms could be need to correct distortions in 3D-space $[15,16]$. 
Depending on the frame rate the imaging length along the web varied from $4.6 \mathrm{~cm}(183 \mathrm{~Hz})$ to $10.0 \mathrm{~cm}(82 \mathrm{~Hz})$. In the Fig. 4 is the 3D-image maximum intensity projection of the surface at $82 \mathrm{~Hz}$. Close to the channel's sidewalls PMMA is thicker (the arrows in Fig. 4b), which is typical for a R2R hot embossing process. Due to a short embossing time, material has not enough time to take a shape of the master mold. This also leads to curved sidewalls instead of rectangular. Fig. 5 shows the surface topographies for all of the frame rates. As could be expected, in the MD direction the lateral step sizes are too large for 82 and $123 \mathrm{~Hz}$ to resolve the bends of the channel (dashed rectangles in Fig. 5a. and 5b.). In addition, the unstable time between the frame acquisitions can make the issue worse. In Fig. 5c., at the $183 \mathrm{~Hz}$ (MD resolution 91 $\mu \mathrm{m})$, the bends are resolved. Although, for accurate determination for the widths and shapes even higher frame rate could be needed. Alternatively, the web speed could be reduce to $0.5 \mathrm{~m} / \mathrm{min}$.

The channel widths were determined using the channel edge-finding algorithm presented in the data processing paragraph. Fig. 5. shows the averaged widths with the standard deviations along the channels depicted the arrows. The average channel widths are consistent with each other and standard deviations are below the CD step size. For the CD step sizes of $10 \mu \mathrm{m}$ and $20 \mu \mathrm{m}$ (Fig. 5a. and 5b.) the averaged widths are the similar. For the $50 \mu \mathrm{m}$ step size in Fig. 5c. (the fastest frame rate), the determined widths are
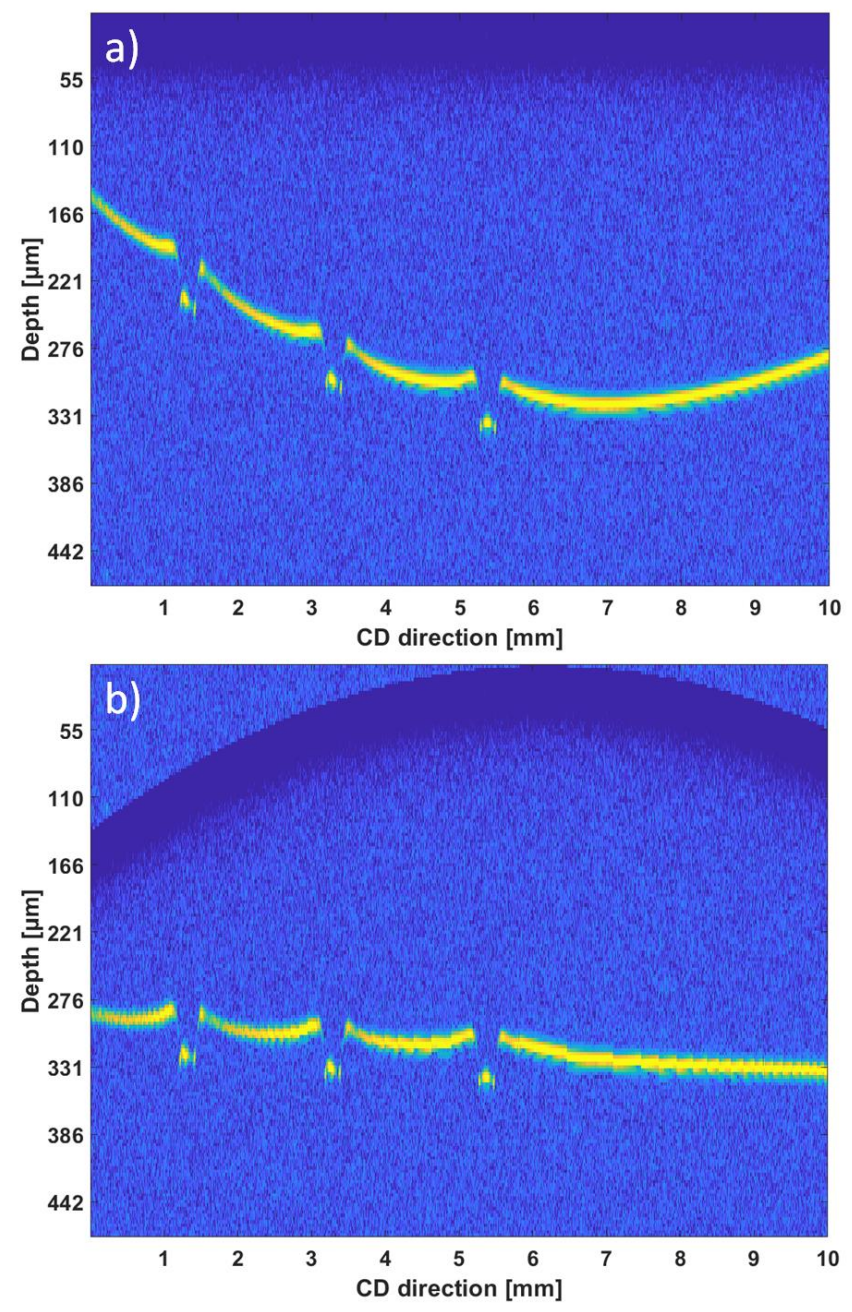

Fig. 3. Correction for a non-telecentric scanning: a) the measured surface and $b$ ) the corrected surface.
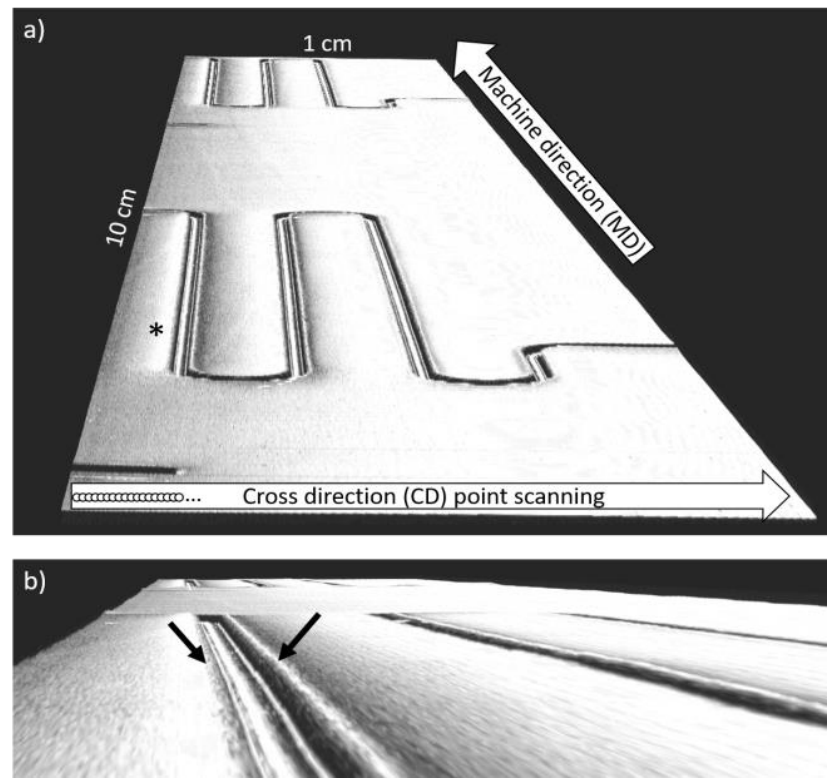

Fig. 4. Maximum intensity projection image of the surface topography measured at the frame rate of $82 \mathrm{~Hz}$. OCT point scanning was done in $\mathrm{CD}$ direction. a) The entire data set and b) the close-up from the channel with the elevated walls (black arrows).

higher, which is due to the poor resolution compared to the channel width of $200 \mu \mathrm{m}$. The channel widths were determined for the $10 \mu \mathrm{m} \mathrm{CD}$ step size at the static conditions to estimate an effect of moving and vibrating web on the channel width determination. Fig. 5d. shows the averaged widths and the standard deviations of the channels when the R2R-line is stopped. The widths are comparable to
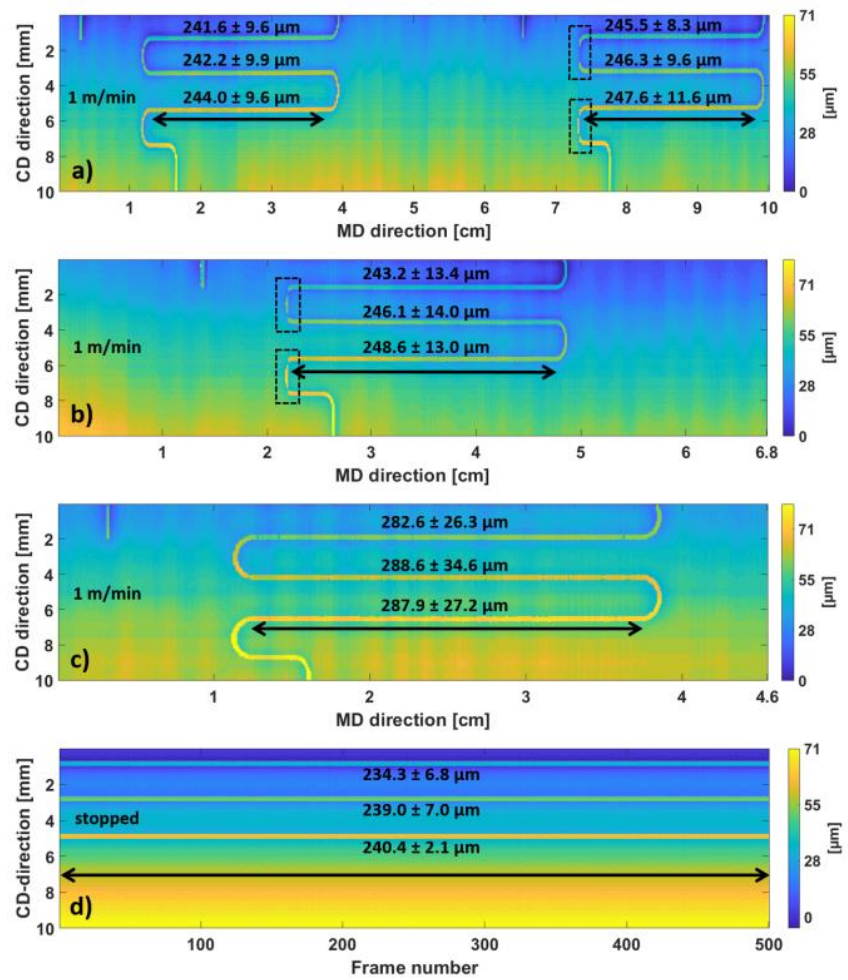

Fig. 5. Surface topography images measured at the web speed of $1 \mathrm{~m} / \mathrm{min}$ and frame rates of a) $82 \mathrm{~Hz}$, b) $123 \mathrm{~Hz}$, c) $183 \mathrm{~Hz}$. d) web was stopped and measured at $82 \mathrm{~Hz}$. The determined averaged widths with the stantard deviations along the arrow are shown. The dashed rectangles indicate poorly resolved bends in the MD direction at $82 \mathrm{~Hz}$ and $123 \mathrm{~Hz}$ frame rates. 

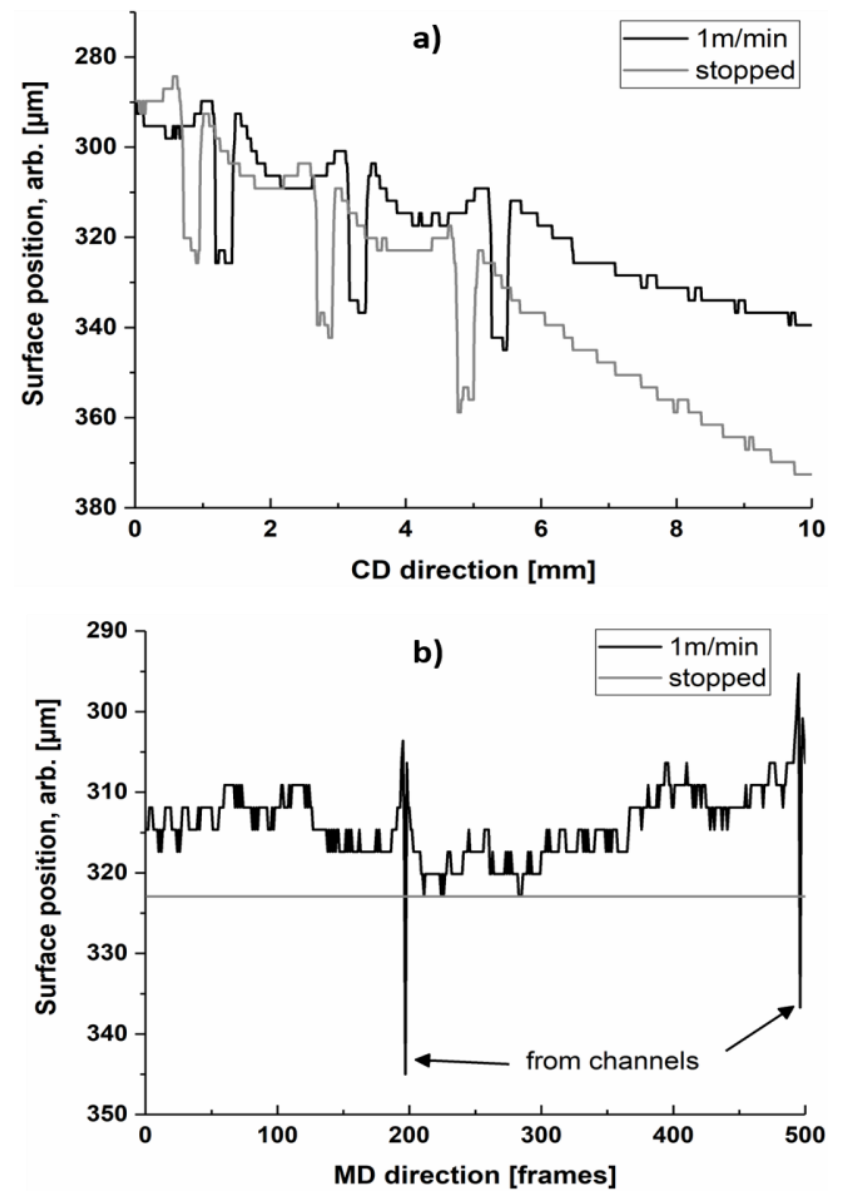

Fig. 6. Surface profiles at the web speed of $1 \mathrm{~m} / \mathrm{min}$ and at stop. a) in the $\mathrm{CD}$ direction and $\mathrm{b}$ ) in the MD direction.

those obtained at the web speed of $1 \mathrm{~m} / \mathrm{min}$, thus, the moving web has only minor influence on the precision.

Vibrations are often an issue in online measurements of micrometer scale features. A level of vibrations caused by a moving web was compared a stopped situation. By comparing the stopped web in Fig. 5d. to the moving ones in Fig. 5a-c., it can be seen that indeed there are lot of vibrations caused by the moving web, although, mostly visibly in MD direction. Fig. 6a. and 6b. show the surface profiles of the stopped and moving web in the $\mathrm{CD}$ and $\mathrm{MD}$ directions respectively. Because of the fast frame rate in $\mathrm{CD}$ direction, vibrations cause no issue in determining channel widths, however, for instance surface roughness measurements are difficult and require additional signal processing or reference measurements for the vibration cancellation. In the MD direction, in motion, the peak-topeak surface vibration was about $15 \mu \mathrm{m}$

The reference channel's shape and width were measured with the optical profilometer, see Fig. 7a. At the steep channel's sidewalls, the optical signal is lost and no data is available, which is also the case for the OCT. For the OCT and automated surface segmentation, the missing data points are causing noise peaks. The peaks, located at the sidewalls, are removed by median filtering. However, the filtering will lead to the increased width $(240 \mu \mathrm{m}$ vs. $200 \mu \mathrm{m})$ of the bottom part of the channel compared to the optical profilometer results. To match the widths, OCT data processing needs to be developed further or to apply a calibration data, which would lead to correct absolute widths.
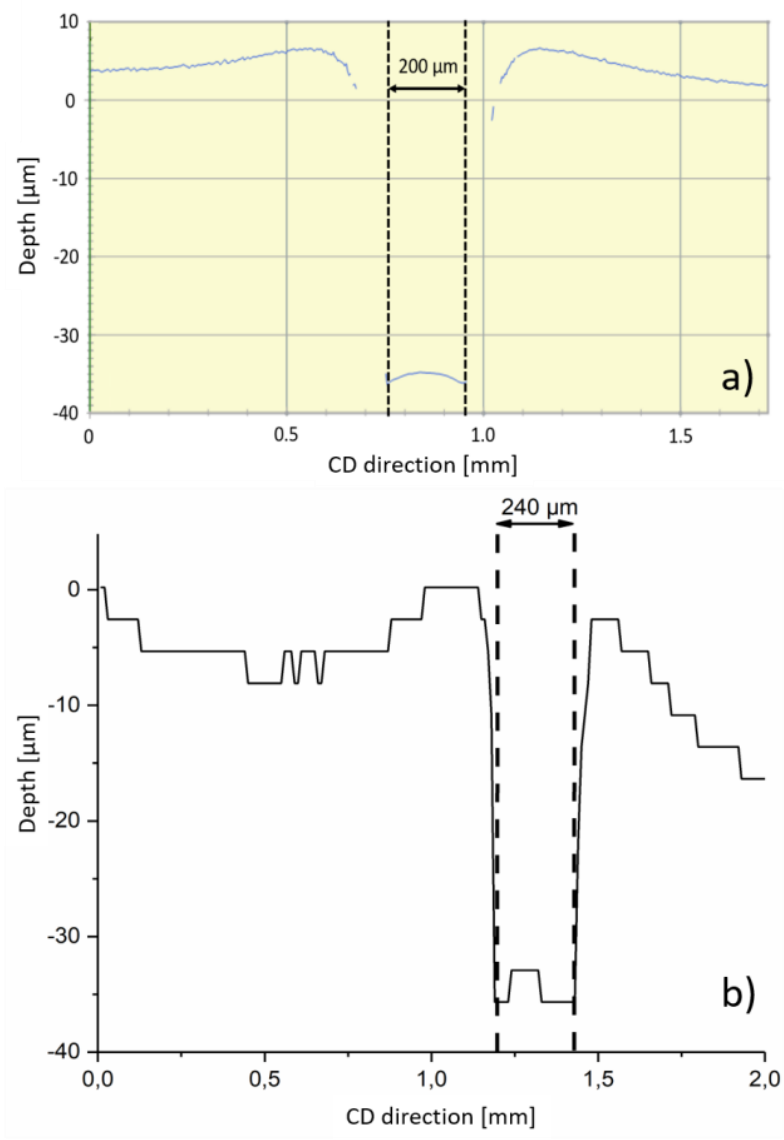

Fig. 7. Channel profiles measured with a) the optical profilometer (ContourGT-K) and b) the OCT. The determined width from the OCT data is higher compared to the reference.

Although, for a process quality control absolute values are often not required. This is because, typically, it is enough to follow trends and to keep parameters, such as the average width or its standard deviation, between set limits. As for the shape, the reference measurement shows similar elevations next to the channel's sidewalls as for the profile measured by OCT (Fig. 7b). The curved bottom part of the channel is also resolved by OCT, although vertical resolution is much worse. Different algorithms, such as Gaussian fitting to the surface intensity data or zero padding before taking a Fourier transform, could be applied to improve depth resolution in surface mapping. To achieve nanometer accuracy, phase mapping techniques can be applied [17]. However, it will increase computation time, which is critical in online measurements. For OCT, the surface appears to go more downwards after the channel than in the reference. This is because of the residual tilt in OCT measurements.

\section{CONCLUSION}

OCT was successfully applied at the R2R-line to measure surface characteristics of the hot embossed microchannels. The widths of the channels were determined by using the automated algorithm and the results were a bit higher $(240 \mu \mathrm{m}$ vs. $200 \mathrm{~mm}$ ) than reference measurements with the optical profilometer. Reasons for the larger widths were due to the OCT's relatively large step sizes in CD direction as well as the median smoothing in the surface segmentation. OCT had problems to resolve bends due to the poor MD resolution. Currently, there are OCT systems with much 
higher imaging speeds available and can improve MD resolution even by a factor of ten.

The shape of the channel was resolved by the OCT, however, parts of the data were lost because of no light could be detected from the steep sidewalls. This was also the case for the optical profilometer used in the reference measurements. In the surface topography measurements, OCT suffered from the poor depth resolution compared to the optical profilometer. However, with different signal processing methods, such as zero padding the camera intensity data before taking a Fourier transform or Gaussian fitting to the intensity data from the surface would lead to much higher surface mapping accuracy. In addition, phase mapping algorithms can be applied to achieve nanometers depth resolution in surface characterization.

As was assumed, there were vibrations caused by the moving web. For the B-scans, taken at the $\mathrm{CD}$ direction, the moving and stopped web appeared to be similar. In the MD direction, the measurement time was much longer and vibrations became more evident. At stop, no vibrations were observed, while in motion, the peak-to-peak surface vibration was about $15 \mu \mathrm{m}$.

As was demonstrated, the OCT is a powerful tool for online quality characterization of the hot embossed microchannel structures in R2R-line. However, its true potential is in 3D structural measurements and evaluation of quality a complete channel structures. For instance resolving quality issues, which occur while lidding channels.

\section{REFERENCES}

[1] I. Yamaguchi, A. Yamamoto and M. Yano, "Surface topography by wavelength scanning interferometry" Optical Engineering 2000, 39, 40-46.

[2] Lédl, V.; Psota, P.; Kaván, F.; Matoušek, O.; Mokrý, P. Surface topography measurement by frequency sweeping digital holography. Appl Opt 2017, 56, 7808-7814, DOI 10.1364/AO.56.007808.

[3] Walecki, W.J.; Szondy, F.; Hilali, M.M. Fast in-line surface topography metrology enabling stress calculation for solar cell manufacturing for throughput in excess of 2000 wafers per hour. Measurement Science and Technology 2008, 19, 025302, DOI 10.1088/0957-0233/19/2/025302.

[4] Molesini, G.; Pedrini, G.; Poggi, P.; Quercioli, F. Focus-wavelength encoded optical profilometer. Opt Commun 1984, 49, 229-233, DOI https://doi.org/10.1016/0030-4018(84)90179-2.
[5] K. Niemelä Chromatic line confocal technology in high-speed 3D surface-imaging applications, Proc. SPIE 10925, Photonic Instrumentation Engineering VI, 1092500 (22 April 2019); https://doi.org/10.1117/12.2507962.

[6] Stifter, D. Beyond biomedicine: a review of alternative applications and developments for optical coherence tomography. Applied Physics B 2007, 88, 337-357, DOI 10.1007/s00340-007-2743-2.

[7] Fercher, A.F.; Drexler, W.; Hitzenberger, C.K.; Lasser, T. Optical coherence tomography - principles and applications. Reports on Progress in Physics 2003, 66, 239.

[8] Lauri, J.; Czajkowski, J.; Myllylä, R.; Fabritius, T. Measuring flow dynamics in a microfluidic chip using optical coherence tomography with $1 \mu \mathrm{m}$ axial resolution. Flow Meas Instrum 2015, 43, 1-5, DOI //dx.doi.org/10.1016/j.flowmeasinst.2015.02.001.

[9] Lauri, J.; Liedert, C.; Kokkonen, A.; Fabritius, T. Effect of solvent lamination on roll-to-roll hot-embossed PMMA microchannels evaluated by optical coherence tomography. Materials Research Express 2019, 6, 075333, DOI 10.1088/2053-1591/ab11a7.

[10] Czajkowski, J.; Lauri, J.; Sliz, R.; Falt, P.; Fabritius, T.; Myllyla, R.; Cense, B. Sub-micron resolution high-speed spectral domain optical coherence tomography in quality inspection for printed electronics, 2012; , pp. 84300-8, DOI //dx.doi.org/10.1117/12.922443.

[11] Kim, S.; Kim, J.; Kang, S. Nondestructive defect inspection for LCDs using optical coherence tomography. Displays 2011, 32, 325-329, DOI https://doi.org/10.1016/j.displa.2011.04.002.

[12] Cho, N.H.; Jung, U.; Kim, S.; Kim, J. Non-Destructive Inspection Methods for LEDs Using Real-Time Displaying Optical Coherence Tomography. Sensors 2012, 12, DOI 10.3390/s120810395.

[13] Alarousu, E.; AlSaggaf, A.; Jabbour, G.E. Online monitoring of printed electronics by Spectral-Domain Optical Coherence Tomography. Scientific Reports 2013, 3, 1562.

[14] Westphal, V.; Rollins, A.M.; Radhakrishnan, S.; Izatt, J.A. Correction of geometric and refractive image distortions in optical coherence tomography applying Fermat's principle. Opt Express 2002, 10, 397 404, DOI 10.1364/OE.10.000397.

[15] M. Ourak; B. Tamadazte; G. J. Laurent; N. Andreff Geometric Calibration of an OCT Imaging System. 2018 IEEE International Conference on Robotics and Automation (ICRA) 2018, 3993-3999, DOI 10.1109/ICRA.2018.8463171.

[16] Ortiz, S.; Siedlecki, D.; Remon, L.; Marcos, S. Optical coherence tomography for quantitative surface topography. Appl Opt 2009, 48, 6708-6715, DOI 10.1364/AO.48.006708.

[17] Das, S.; Liu, C.; Singh, M.; Twa, M.D.; Larin, K.V. Modified wavelength scanning interferometry for simultaneous tomography and topography of the cornea with Fourier domain optical coherence tomography. Biomedical optics express 2018, 9, 4443-4458, DOI 10.1364/BOE.9.004443. 